\title{
Myocardial strains from 3D displacement encoded magnetic resonance imaging
}

\author{
Katarina Kindberg ${ }^{1,2 \dagger}$, Henrik Haraldsson ${ }^{1,2,3^{*}+}$, Andreas Sigfridsson ${ }^{2,3}$, Jan Engvall ${ }^{3}$, Neil B Ingels Jr $\mathrm{r}^{4,5}$, \\ Tino Ebbers ${ }^{1,2,3,6}$ and Matts Karlsson ${ }^{1,2}$
}

\begin{abstract}
Background: The ability to measure and quantify myocardial motion and deformation provides a useful tool to assist in the diagnosis, prognosis and management of heart disease. The recent development of magnetic resonance imaging methods, such as harmonic phase analysis of tagging and displacement encoding with stimulated echoes (DENSE), make detailed non-invasive 3D kinematic analyses of human myocardium possible in the clinic and for research purposes. A robust analysis method is required, however.

Methods: We propose to estimate strain using a polynomial function which produces local models of the displacement field obtained with DENSE. Given a specific polynomial order, the model is obtained as the least squares fit of the acquired displacement field. These local models are subsequently used to produce estimates of the full strain tensor.

Results: The proposed method is evaluated on a numerical phantom as well as in vivo on a healthy human heart. The evaluation showed that the proposed method produced accurate results and showed low sensitivity to noise in the numerical phantom. The method was also demonstrated in vivo by assessment of the full strain tensor and to resolve transmural strain variations.

Conclusions: Strain estimation within a 3D myocardial volume based on polynomial functions yields accurate and robust results when validated on an analytical model. The polynomial field is capable of resolving the measured material positions from the in vivo data, and the obtained in vivo strains values agree with previously reported myocardial strains in normal human hearts.
\end{abstract}

\section{Background}

The pumping behavior of the heart consists of complex sequences that constitute cardiac contraction and relaxation. The kinematic behavior of the heart has been analyzed extensively in order to understand the mechanisms that impair the contractile function of the heart during disease. Until recently, the only method with high enough spatial resolution of three-dimensional (3D) myocardial displacements to resolve transmural behaviors was invasive marker technology $[1,2]$. However, the recent development of magnetic resonance imaging (MRI) methods such as harmonic phase (HARP) analysis of tagging [3] and displacement

\footnotetext{
* Correspondence: Henrik.Haraldsson@liu.se

† Contributed equally

'Department of Management and Engineering, Linköping University, SE-581

83 Linköping, Sweden

Full list of author information is available at the end of the article
}

encoding with stimulated echoes (DENSE) [4], make detailed non-invasive 3D transmural kinematic analyses of human myocardium possible for clinical and research purposes [5].

A previously presented polynomial method for cardiac strain quantification from surgically implanted markers and beads enables straightforward 3D strain computation within the myocardium [6]. For the marker and bead data, this method was shown to yield accurate and robust results, with errors smaller or comparable to a previously presented finite element method tailored for the same type of data, and has been applied to bead displacements for analyses of systolic and diastolic myocardial strains [7-9]. The method is simple in nature which aids to bridge a possible gap in understanding between different disciplines and has specifically been shown to be well suited for sparse arrays of displacement data [6]. The present work applies this polynomial method to 3D

\section{Biomed Central}


MRI displacement data as it: 1) allows for quantification of the full 3D strain tensor; 2) resolves transmural strain variations within the myocardium, and 3) provide robustness to noise. Displacement measurements were acquired within a 3D myocardial slab at the left ventricular (LV) equator. The 3D volume was divided into segments where the polynomial method was applied to quantify the Lagrangian strain tensor. The method was evaluated on a numerical phantom as well as in vivo on a healthy human heart.

\section{Methods}

\section{Strain analysis}

Displacement-encoded MRI acquire displacements $\mathbf{u}$ at time $t_{n}$ of timeframe $n$ relative to a reference configuration of the myocardium, at the spatial coordinates $\mathbf{s}=$ $\left(s_{x}, s_{y}, s_{z}\right)$. Lower-case letters are used to denote the coordinates of the deformed myocardium. The spatial coordinates $\mathbf{S}=\left(\mathrm{S}_{\mathrm{X}}, \mathrm{S}_{\mathrm{Y}}, \mathrm{S}_{\mathrm{Z}}\right)$ of the myocardium in the reference configuration were derived by subtracting the displacements from the spatial coordinates at the current configuration; $\mathbf{S}\left(\mathrm{t}_{1}\right)=\mathbf{s}-\mathbf{u}\left(\mathbf{s}, \mathrm{t}_{1}\right)$. The upper-case letters are used to denote the coordinates of the reference configuration.

$\mathbf{X}=\mathbf{X}(\mathbf{S})$ is the coordinate of a material point, defined as an infinitesimal volume of myocardial tissue, in the reference configuration and $\mathbf{x}=\mathbf{x}(\mathbf{s})$ is the coordinate after a deformation of the body. The mapping from reference to deformed configuration was modeled by a polynomial function $\mathbf{g}(\mathbf{X})$ of the coordinate of the material point in the reference configuration [6]. This polynomial position field gives an estimate $\hat{\mathbf{x}}$ of the measured coordinate $\mathbf{x}$ and can be of different order in the different reference coordinate directions, radial $\left(X_{R}\right)$, circumferential $\left(\mathrm{X}_{\mathrm{C}}\right)$, and longitudinal $\left(\mathrm{X}_{\mathrm{L}}\right)$, depending on the number of material points along each dimension, and can thus be described as

$$
\hat{\mathbf{x}}=\mathbf{g}(X)=f_{R}\left(X_{R}\right) f_{C}\left(X_{C}\right) f_{L}\left(X_{L}\right),
$$

where $\mathbf{f}_{R}, \mathbf{f}_{C}$ and $\mathbf{f}_{L}$ are polynomial functions of $X_{R}, X_{C}$ and $\mathrm{X}_{\mathrm{L}}$, respectively. The Lagrangian strain tensor $\mathbf{E}$ is a measure of deformation and is connected to the deformation gradient tensor $\mathbf{F}$ via the definition

$$
\mathbf{E}=0.5\left(\mathbf{F}^{\mathrm{T}} \mathbf{F}-\mathbf{I}\right)
$$

where $\mathbf{I}$ is the identity tensor. The deformation gradient tensor is given by differentiation, with respect to reference position, of the mapping from reference to deformed configuration, $\mathbf{F}=\partial \mathbf{x} / \partial \mathbf{X}$.

The principal strains $E_{1}, E_{2}$ and $E_{3}$ are obtained by diagonalization of the strain tensor, and their magnitudes are independent of any reference coordinate system. Principal strain $\mathrm{E}_{1}$ represents maximum lengthening and $\mathrm{E}_{3}$ represents maximum shortening.

In the subsequent analytical and in vivo evaluation, the $3 \mathrm{D}$ volumes were divided into 80 overlapping segments covering the circumference. Each of the segments was $7.5 \mathrm{~mm}$ thick and $\pi / 6$ radians wide between the endo- and epicardial border. Within each segment the material points were transformed to local Cartesian cardiac coordinates with $\mathrm{X}_{\mathrm{C}}$ defined as tangential to the surface of the volume and parallel with the short axis planes, $\mathrm{X}_{\mathrm{L}}$ defined as orthogonal to the short axis planes and oriented apex-to-base, and $X_{R}$ defined parallel with the short axis planes, orthogonal to $\mathrm{X}_{\mathrm{C}}$ and oriented outwards from the volume.

Four orders of the polynomial function $\mathbf{g}(\mathbf{X})$ were analyzed. 1) A bilinear-quadratic polynomial (BLQ) was bilinear within the $X_{C}-X_{L}$ plane and quadratic in $X_{R}, 2$ ) a bilinear-cubic polynomial (BLC) was bilinear within the $\mathrm{X}_{\mathrm{C}}-\mathrm{X}_{\mathrm{L}}$ plane and cubic in $\mathrm{X}_{\mathrm{R}}, 3$ ) a linear-quadraticcubic polynomial (LQC) was cubic in $X_{R}$, quadratic in $\mathrm{X}_{\mathrm{C}}$ and linear in $\mathrm{X}_{\mathrm{L}}$ and 4) a biquadratic-cubic polynomial (BQC) was biquadratic within the $\mathrm{X}_{\mathrm{C}}-\mathrm{X}_{\mathrm{L}}$ plane and cubic in $\mathrm{X}_{\mathrm{R}}$. For example, for the LQC polynomial field, the estimated coordinates were given by

$$
\begin{aligned}
& \hat{x}_{i}=\left(a_{1 i} X_{R}^{3}+a_{2 i} X_{R}^{2}+a_{3 i} X_{R}+a_{4 i}\right)\left(a_{5 i} X_{C}^{2}+a_{6 i} X_{C}+a_{7 i}\right)\left(a_{8 i} X_{L}+a_{9 i}\right) \\
& =\left[X_{R}^{3} X_{C}^{2} X_{L}, \ldots, 1\right] \cdot\left[\begin{array}{c}
c_{1 i} \\
\vdots \\
c_{24 i}
\end{array}\right]
\end{aligned}
$$

where $a_{k i}$ and $c_{l i}$ are sets of constants, $i=R, C, L$, and $k$ and $l$ are serial numbers for the indices. The polynomial field for each segment was determined by minimizing the squared difference between the measured and the estimated coordinates of the material points belonging to the segmentation.

$$
\min _{\mathbf{c}_{i}} \sum_{j=1}^{m}\left(x_{i j}-\hat{x}_{i j}\left(\mathbf{c}_{i}\right)\right)^{2},
$$

where $m$ is the total number of points within the segment and $c_{i}$ are all the constants $c_{l i}$. The polynomial was evaluated and the Lagrangian strain tensor quantified at material positions along the radial centerline within each segment. The accuracy of the polynomial function was evaluated by the residual of the polynomial mapping, determined as the root-mean-squared difference (RMS) between the measured ( $\mathbf{x})$ and estimated $(\hat{\mathbf{x}})$ coordinates within each segment. The strain analysis was performed off line using Matlab 7.6.0 (The MathWorks, Inc.). 


\section{Analytical evaluation}

A previously presented analytical model $[6,10]$ was used to evaluate the strain computation. Briefly, the model was a thick-walled cylinder that deforms to resemble the LV during systole. The cylinder was adapted to the systolic in vivo data with inner radius of $28.5 \mathrm{~mm}$ and outer radius of $42.4 \mathrm{~mm}$ at the reference configuration, and inner radius of $23.6 \mathrm{~mm}$ at the deformed configuration. Material points were sampled through the cylinder wall within ten short axis planes with $2.5 \mathrm{~mm}$ separation between the planes and $2.5 \times 2.5 \mathrm{~mm}$ separation between sampled points within each plane.

Strains were computed throughout the model using the method described above. For all polynomial orders, the estimated strains were compared with the analytical strains $\left(\mathbb{\Xi}_{I J}\right.$, where $\left.I, J=R, C, L\right)$. To mimic the acquisition of in vivo data, noise was added to the analytical model to investigate the noise sensitivity of the analysis method.

Three normalized distributions of noise were analyzed, each with a mean value of zero and with the standard deviations $0.05 \mathrm{~mm}, 0.10 \mathrm{~mm}$ and $0.30 \mathrm{~mm}$, respectively. The noise levels evaluated were chosen to represent practical measurement errors in DENSE displacement measurements corresponding to SNR in the order of 35 , 17 and 6.

\section{In vivo evaluation}

In vivo evaluation was performed by applying the strain quantification method to displacements acquired by DENSE MRI in a normal human heart, and comparing the results with strains obtained for normal human hearts from other studies.

Anatomical images and displacement data were acquired in a 31-year old healthy volunteer on a $1.5 \mathrm{~T}$ MR scanner (Philips Achieva, Philips Medical Systems, Best, The Netherlands). Displacement data were acquired, using an in-house DENSE implementation, in three cardiac phases as illustrated in Figure 1; one in systole, and two during diastole related to early and late filling. The cardiac phases were defined from balanced steady state free precession acquisition in a standard 3 chamber view with a temporal resolution of $13 \mathrm{~ms}$. These images were used to define mitral valve opening (MVO) and end systole (ES), defined as aortic valve closure (AVC). These two events were used to time the measurement of displacement in systole and diastole, acquired in 3D short axis slab in two separate 3D DENSE acquisitions. For the systolic measurement, the initial reference position was encoded at end diastole (ED), defined by the ECG R-wave peak, and the displacement was read out at ES, as shown in Figure 1. For the displacement in diastole, the initial reference position was encoded at MVO and read out at both MVO +

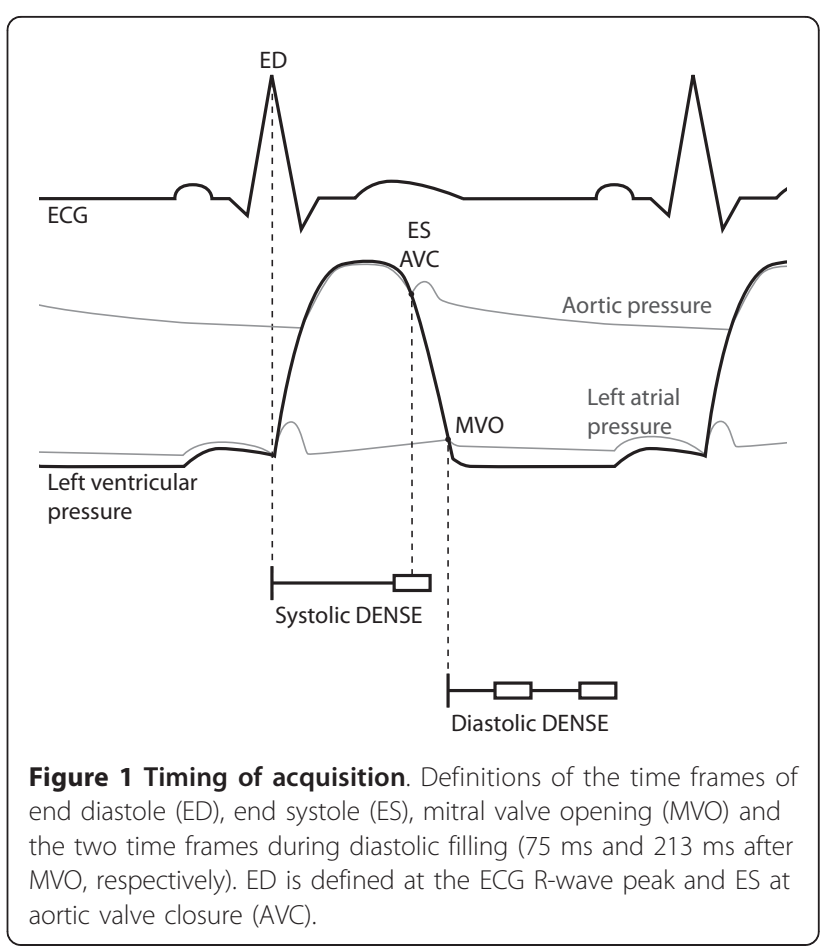

$75 \mathrm{~ms}$ and at $\mathrm{MVO}+213 \mathrm{~ms}$, which corresponds to approximately $22 \%$ and $62 \%$, respectively, of the LV filling interval. The 3D DENSE acquisitions were performed with an ECG triggered, respiratory navigator gated acquisition with the following parameters: acquisition voxel size $2.5 \times 2.5 \times 2.5 \mathrm{~mm}$, field of view $350 \times$ $350 \times 25 \mathrm{~mm}, k$-space segmentation factor 3, EPI factor 7 , parallel imaging (SENSE) with reduction factor 2 , echo time $5.7 \mathrm{~ms}$, repetition time $12.2 \mathrm{~ms}$, balanced multi-point displacement encoding 0.35 cycles per pixel [11], 3-SPAMM [12] displacement encoding with optimized flip angle [13]. The sequence requires data acquisition of 346 heart beats. Including navigator gating, the scan time is in the order of 10-15 minutes, depending on heart rate and navigator efficiency. For this DENSE sequence, a noise level of about $0.05 \mathrm{~mm}$ can be expected for a typical in-vivo measurement.

Segmentation of the myocardium was performed using the freely available software Segment [14]. To avoid that non-myocardial tissue could affect the myocardial strain estimates, the segmentation was conservative, excluding uncertain areas or voxels. The phase of the DENSE MRI is proportional to the myocardial displacement, but can be an arithmetic modulo $2 \pi$, which can be represented by wrapping the phase into the interval $-\pi$ to $\pi$. Using the segmentation to outline the myocardium, the MRI phase was unwrapped [15] using a multigrid solver to the Poisson equations [16].

Systolic Lagrangian strains were analyzed at ES, with reference configuration at ED. Diastolic Lagrangian 
strains were analyzed during LV filling with reference configuration at MVO.

The research has been performed with informed consent, approved by the Regional Ethical Review Board in Linköping and carried out in compliance with the Helsinki Declaration.

\section{Results}

\section{Analytical evaluation}

The size of the errors of estimated strains is dependent on the spatial resolution of the sampled displacements [6]. Given the analytical strain components $E_{I J}$ of the model, the absolute errors of the estimated strains in the analytical model, $\varepsilon_{I J}=\left|\bigotimes_{I J}-E_{I J}\right|$, were computed for each polynomial configuration order, integrated throughout each plane and averaged over the planes. For the noise free case, the mean \pm SD error for the strain components of each polynomial order were BLQ: $0.0098 \pm 0.0067$, BLC: $0.0125 \pm 0.0092$, LQC: $0.0068 \pm$ 0.0016 , BQC: $0.0070 \pm 0.0017$. The errors for each component, respectively, from the linear-quadratic-cubic polynomial, for which the smallest mean errors were obtained, were $\varepsilon_{\mathrm{RR}}=0.0081$ for the radial strain, $\varepsilon_{\mathrm{CC}}=$ $0.0054, \varepsilon_{\mathrm{LL}}=0.0089, \varepsilon_{\mathrm{RC}}=0.0072, \varepsilon_{\mathrm{RL}}=0.0048$ and $\varepsilon_{\mathrm{CL}}$ $=0.0067$. The LQC RMS differences, i.e. the residual of the polynomial mapping, averaged within the analytical model, were $\mathrm{RMSx}_{\mathrm{R}}=0.030 \mathrm{~mm}$ in the radial direction, $\mathrm{RMSx}_{\mathrm{C}}=0.017 \mathrm{~mm}$ in the circumferential direction, and $\mathrm{RMSx}_{\mathrm{L}}=0.0004 \mathrm{~mm}$ in the longitudinal direction. The sensitivities to noise for each polynomial are plotted in Figure 2. The LQC polynomial yielded the smallest mean errors in the ideal situation and for a simulated noise with a standard deviation of $0.05 \mathrm{~mm}$ while the BLQ polynomial yielded the smallest mean error for the

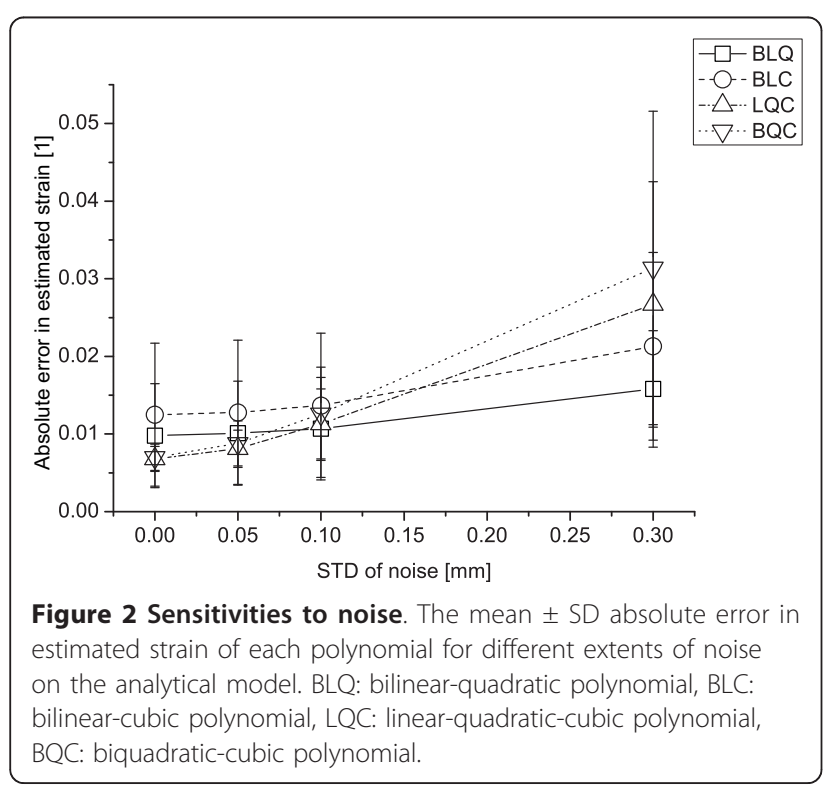

cases with 0.10 and $0.30 \mathrm{~mm}$ standard deviation of noise.

\section{In vivo evaluation}

The LQC and the BQC polynomials yielded the smallest maximum RMS differences in both systole and diastole. The results from the LQC polynomial are analyzed in further detail below. A mid-section (comprised of the short axis slices 5-7) is shown in Figure 3, 4, 5 and 6.

\section{Systole}

The systolic strain components estimated using the polynomial method are shown in Figure 3. For comparison, Figure 4 shows the corresponding components estimated using a finite element method [17].

Maximum lengthening (up to 0.74) was found in the subendocardium in the septum and lateral free wall. Maximum shortening (down to -0.35) was found in the subendocardium and was essentially evenly distributed throughout the plane.

For all three directions, the lowest RMS values were found in septum and the highest values in the posterior wall. $\mathrm{RMSx}_{\mathrm{R}}$ was within the range $0.13-0.40 \mathrm{~mm}$, $\mathrm{RMSx}_{\mathrm{C}} 0.09-0.21 \mathrm{~mm}$, and RMSx $\mathrm{L}_{\mathrm{L}} 0.09-0.24 \mathrm{~mm}$.

\section{Diastole}

All diastolic strain components at $75 \mathrm{~ms}$ (22\% of the filling interval) and $213 \mathrm{~ms}$ (62\%) after MVO at the midsection of the 3D volume are shown in Figure 5.

The diastolic principal strains at $213 \mathrm{~ms}$ after MVO at the mid-section of the 3D volume are shown in Figure 6. Maximum lengthening (up to 0.35) was found in the postero-lateral wall while maximum shortening was essentially evenly distributed over the plane.

The highest RMS values were found in the posterior wall at $75 \mathrm{~ms}$ after MVO and in the anterior and posterior walls at $213 \mathrm{~ms}$ after MVO. The RMS values ranged from $0.07 \mathrm{~mm}$ to $0.40 \mathrm{~mm}$ for all directions at both diastolic times, except RMSx $x_{R}$ at $75 \mathrm{~ms}$ after MVO which approached $0.50 \mathrm{~mm}$ in a small region in the posterior wall.

\section{Discussion}

The proposed myocardial strain quantification method was initially developed for strain computation on data from a surgically implanted transmural bead array. However, this work shows that the method can be extended to be used with displacement data from displacement-encoded MRI.

This work uses a polynomial function to find a differentiable expression from the discrete displacement field. This polynomial function assumes continuous displacement in the myocardium, which reflects the connective properties of the myocardial tissue. This a priori 


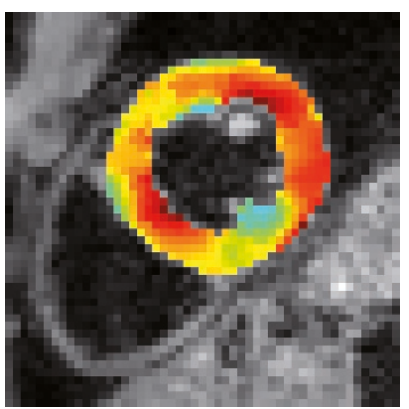

ERR

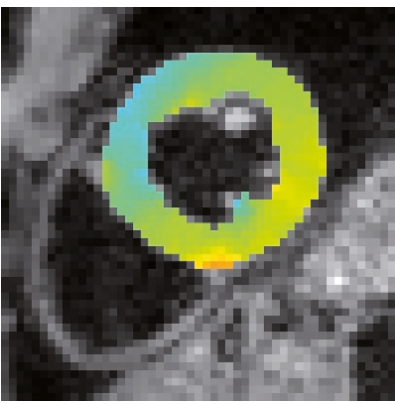

ERC

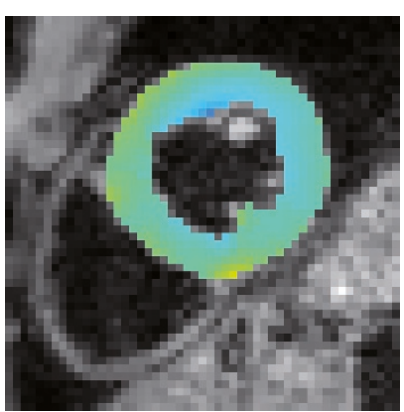

Ecc

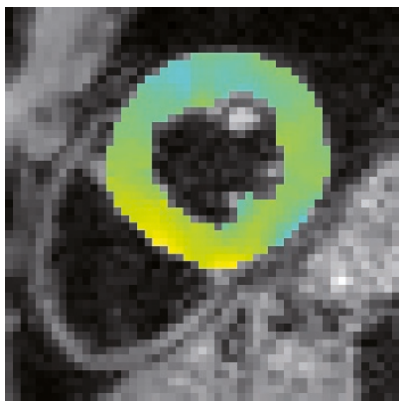

ERL

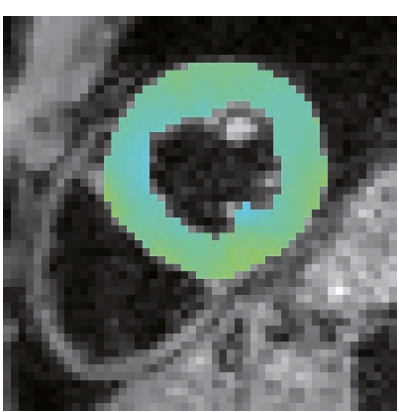

ELL

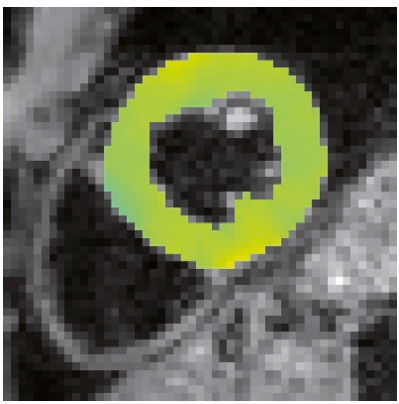

$\mathrm{ECL}$

Figure 3 Systolic strains. All components of the end-systolic strain tensor at the mid-section of the 3D volume in a healthy volunteer estimated using the polynomial method.

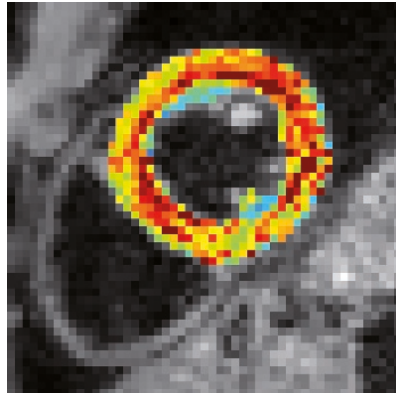

ERR

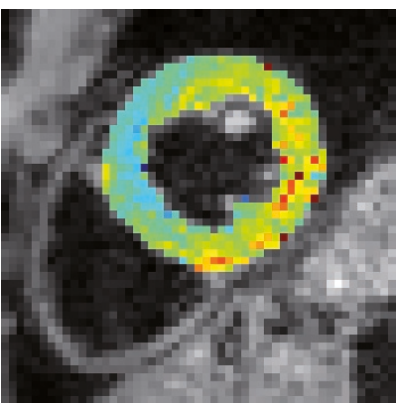

ERC

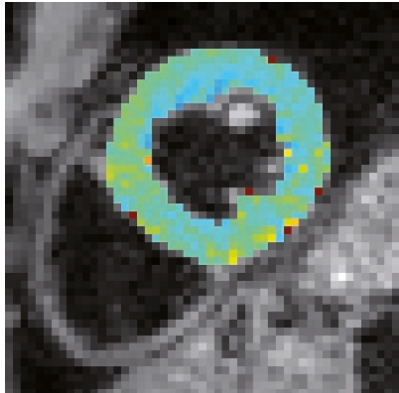

Ecc

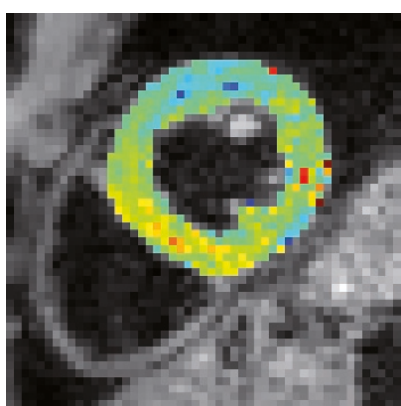

ERL

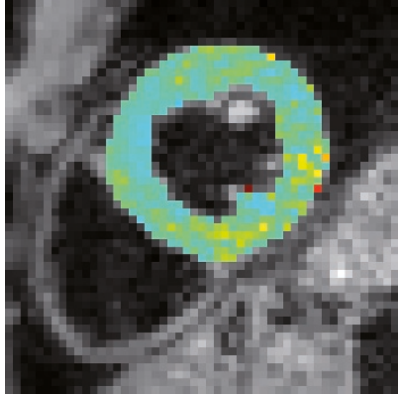

ELL
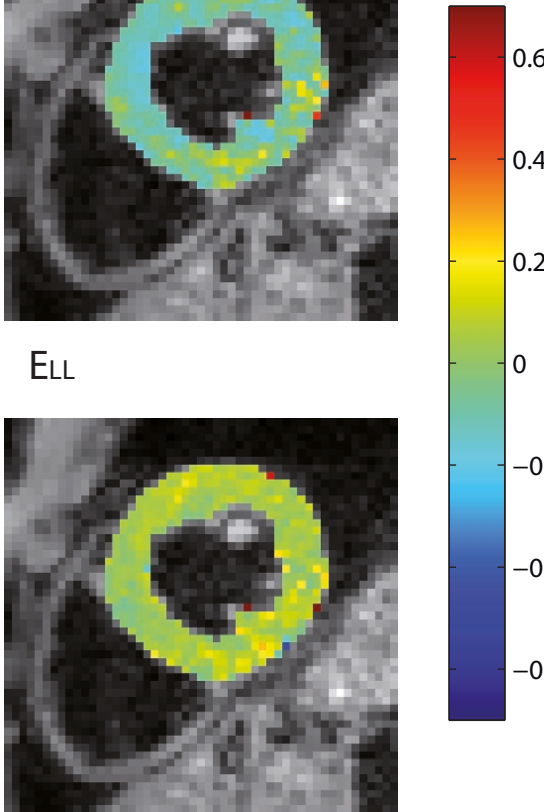

$\mathrm{ECL}$

Figure 4 Systolic strains. All components of the end-systolic strain tensor at the mid-section of the 3D volume in a healthy volunteer estimated using a finite element method. 


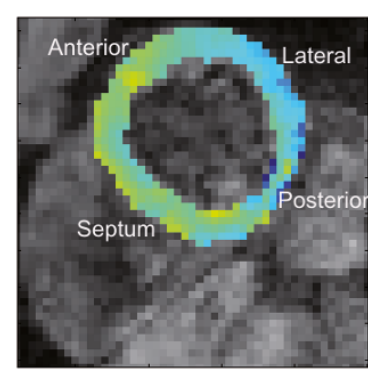

ERR

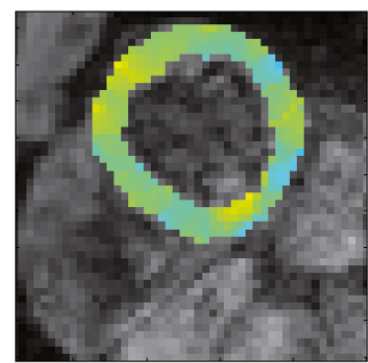

ERC

a) $\mathrm{MVO}+75 \mathrm{~ms}$

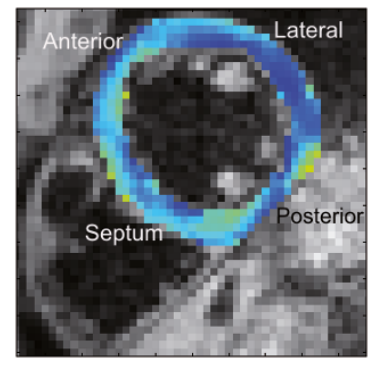

ERR

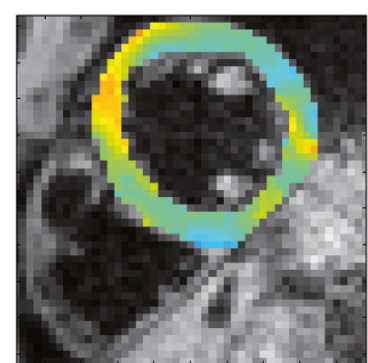

ERC

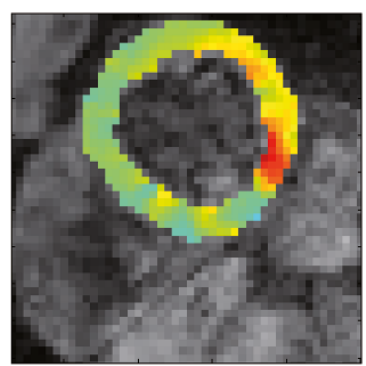

Ecc

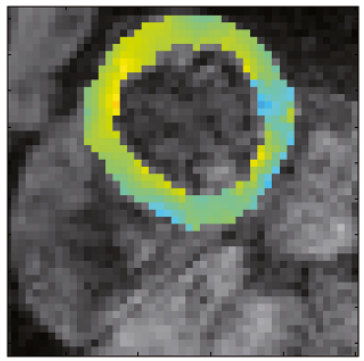

ERL

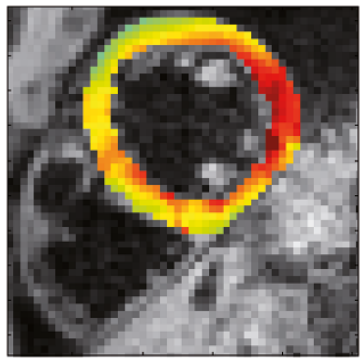

Ecc

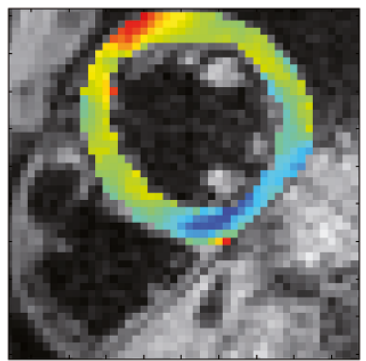

ERL

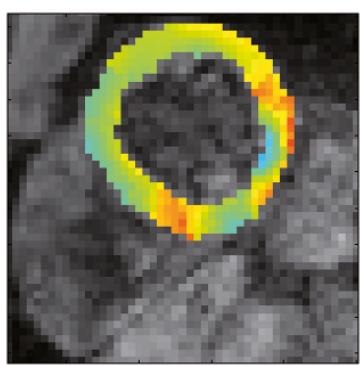

ELL

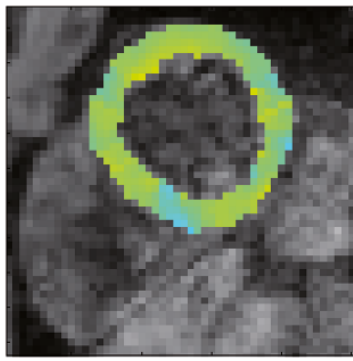

$\mathrm{ECL}$
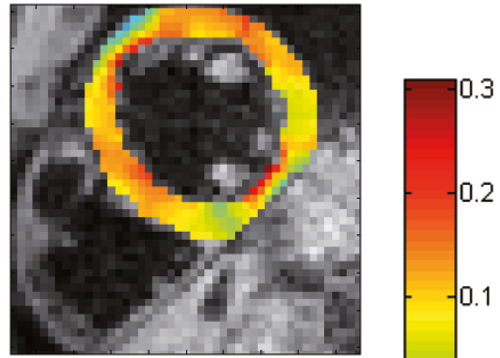

ELL
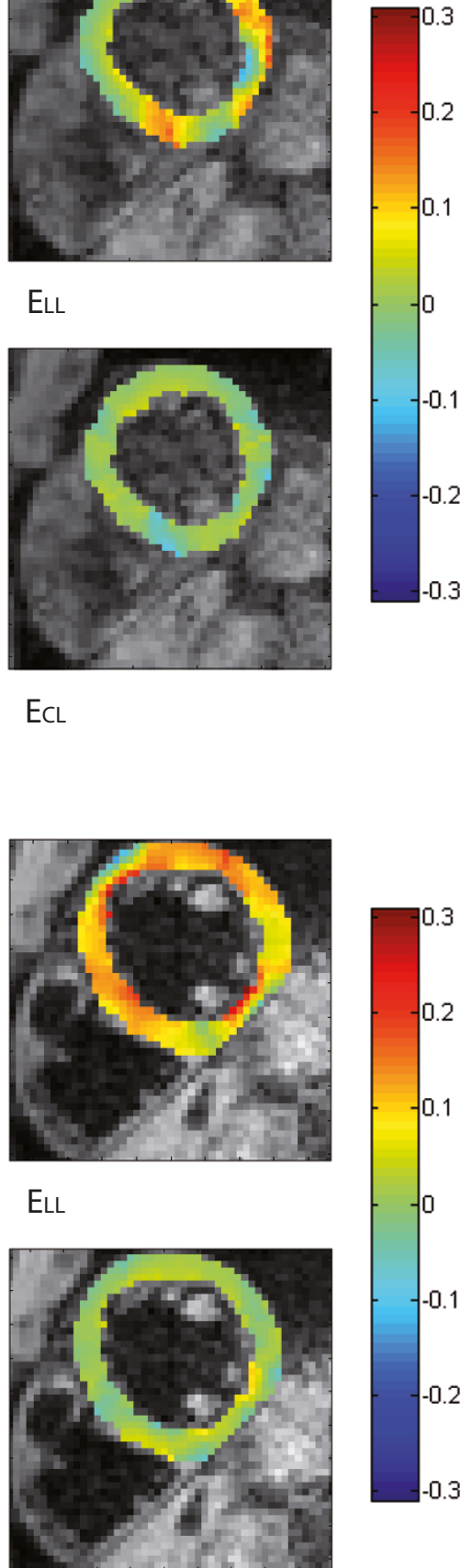

$-0.3$

$\mathrm{ECL}$

b) $\mathrm{MVO}+213 \mathrm{~ms}$

Figure 5 Diastolic strains. All components of the diastolic Lagrangian strain tensor at the mid-section of the 3D volume in a healthy volunteer. a) At 75 ms after MVO; b) At 213 ms after MVO.

information helps making the estimation more robust to noise.

The optimal order of the polynomial functions in equation (1) depends on the number of material points along each dimension, which in turn depends on the spatial resolution of the data, wall thickness and the sizes of the finite segments. Generally, the number of unknown constants in the polynomial should be less 


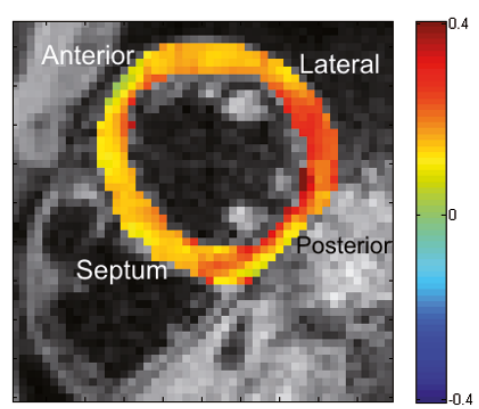

a) $E_{1}$

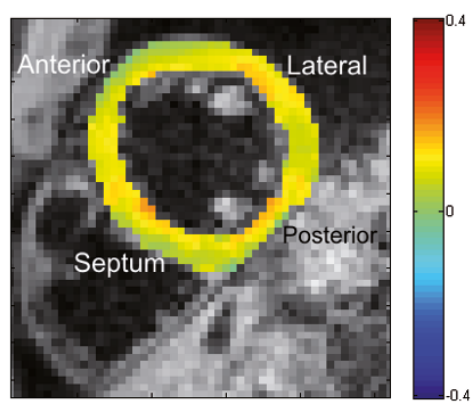

b) $E_{2}$

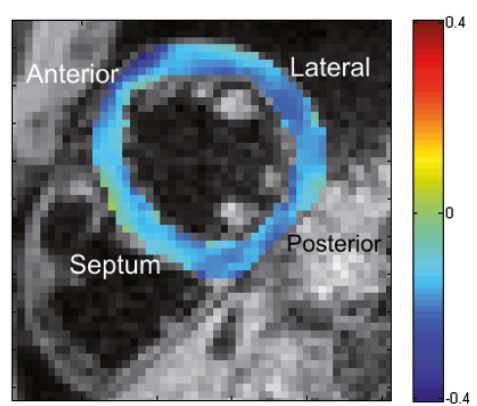

c) $\mathrm{E}_{3}$

Figure 6 Diastolic principal strains. Diastolic principal strains at the mid-section of the 3D volume in a healthy volunteer, at 213 ms after MVO. a) $E 1$; b) E2; c) E3.

than the number of measured points along each dimension, which implies that a third order polynomial requires at least five measured material points along the corresponding dimension, a second order polynomial requires at least four points and a first order polynomial requires at least three points. Furthermore, to avoid an undetermined problem, the number of data points must be equal or greater than the number of coefficients in the minimization. This implies that the minimum number of required data points depends on the polynomial orders; BLQ requires 12 data point, BLC 16, LQC 24, and BQC 36. Hence, the polynomial order is limited by the number of included data points. This requirement was fulfilled for the in vivo evaluation; however, the margin for the BQC polynomial was small at some locations of the myocardium.

Four different polynomial orders were evaluated. The smallest absolute errors of the estimated strains in the analytical model in the presence of low noise were obtained with a linear-quadratic-cubic polynomial. In the subsequent in vivo validation, the in vivo results of the linear-quadratic-cubic polynomial were analyzed in detail. Given the incumbent spatial resolution, the restriction on $\mathbf{f}_{R}\left(X_{R}\right)$ was the wall thickness at the late diastolic time frame and the restrictions on $\mathbf{f}_{\mathrm{C}}\left(\mathrm{X}_{\mathrm{C}}\right)$ and $\mathbf{f}_{\mathrm{L}}\left(\mathrm{X}_{\mathrm{L}}\right)$ were the width and height of each segment, respectively. The width ( $\pi / 6$ radians) and height (7.5 $\mathrm{mm}$ ) of each segment were kept small in order to resolve local variations of deformation.

The RMS differences between the acquired in vivo coordinates and the coordinates estimated using the polynomial fitting method reflect the accuracy of the polynomial fitting, giving a comprehension of the extent to which the coordinates estimated by the polynomial fit to the acquired in vivo coordinates. For both systolic and diastolic data, the RMS differences of the LQC polynomial were highest in the posterior wall. For the systolic data the region of highest RMS differences was close to the posterior papillary muscle, which might have caused locally irregular displacements. The RMS differences may furthermore be affected by the spatially varying signal-to-noise ratio in the DENSE measurement [18]. For the diastolic data, the region of highest RMS differences coincided with the region of thinnest wall. The present implementation of the method used the same polynomial order for each segment within the 3D volume. If a data set with large variations in wall thickness is considered, e.g. a 3D slab comprising an infarct with thin wall near the infarcted myocardium, the highest accuracy could be obtained by local adjustment of the size of the segments to the size of the infarcted region and adapting the polynomial order to wall thickness and segment width.

Systolic radial, circumferential and longitudinal strains, as well as systolic circumferential-longitudinal shear, show agreement with systolic strains previously reported for human myocardium $[19,20]$. $\mathrm{E}_{\mathrm{RC}}$ : We observed somewhat higher magnitudes of radial-circumferential shear strain than the results of Moore et al. [19], and we observed the lowest values in the anterior region of the myocardium while Moore et al. reported the lowest values in septum. $\mathrm{E}_{\mathrm{RL}}$ : The observed radial-longitudinal shear values fits within mean $\pm 2 \mathrm{SD}$ of the values reported by Moore et al. [19].

Diastolic function of the LV is determined by a complex sequence of many interrelated events and parameters including active relaxation, elastic recoil, passive filling characteristics, heart rate and inotropic state. Diastolic LV filling is a highly dynamic process with early and late transmitral inflows. Thus a detailed analysis of myocardial strain during diastole requires resolving the temporal process of diastolic filling.

The highest values of circumferential strain during the first $213 \mathrm{~ms}$ of diastolic filling were observed in the postero-lateral wall. The same quantitative behavior has been reported in previous studies of early diastolic 
strains in normal human hearts [21,22]. Radial strain, interpreted as wall thinning during diastole, was most apparent in the lateral wall, which also has been reported by others [21].

\section{Limitations}

This work is limited to study the kinematics of the heart, focusing on strain. Strain should preferably be related to an unloaded, stress free reference configuration. Using in vivo data, there is no unloaded, stress free configuration of the heart. Instead, the reference configurations correspond to defined time points in the cardiac cycle. The strain presented in this, and similar articles within the field, therefore disregards the residual strain needed to study cardiac kinetics as opposed to kinematics.

In vivo validation of strain is challenging, which is why an analytical model was included in the validation. The analytical model can however never fully describe the cardiac kinematics. For in vivo estimation, the quality of the strain measurements is highly dependent on the quality of the underlying displacement data. While the polynomial fit reduces sensitivity-to-noise in the measurements, image artifacts or errors in the displacement measurements can deteriorate the strain estimates. Improving the DENSE acquisitions is an active field in the MRI research community, and strain analysis methods like the one presented in this paper will benefit directly from such improvements.

\section{Conclusions}

In conclusion, the proposed method for strain estimation within a 3D myocardial volume yields accurate results when validated on an analytical model. The polynomial field is capable of resolving the measured material positions from the in vivo data, and the obtained in vivo strains agree with previously reported myocardial strains in normal human hearts.

\section{Acknowledgements}

The study was supported by grants from Swedish Heart-Lung Foundation and Swedish Research Council.

\section{Author details \\ 'Department of Management and Engineering, Linköping University, SE-581 83 Linköping, Sweden. ${ }^{2}$ Center for Medical Image Science and Visualization (CMIV), Linköping University, SE-581 85 Linköping, Sweden. ${ }^{3}$ Department of Medical and Health Sciences, Linköping University, SE-581 85 Linköping, Sweden. ${ }^{4}$ Department of Cardiothoracic Surgery, School of Medicine, Stanford University, Stanford, CA 94305, USA. ${ }^{5}$ Research Institute of the Palo Alto Medical Foundation, Palo Alto, CA 94305, USA. ${ }^{6}$ Department of Science and Technology, Linköping University, SE-581 83 Linköping, Sweden.}

\section{Authors' contributions}

KK developed the analysis method, participated in the study design, carried out the analysis of the data, participated and coordinated the writing of the manuscript. $\mathrm{HH}$ participated in the study design, participated in the design of the data acquisition, participated in writing the manuscript. AS participated in the design of the data acquisition, participated in writing the manuscript. JE participated in the design of the data acquisition. NBI participated in the development of the analysis method, participated in the writing of the manuscript. TE participated in the study design, participated in the design of the data acquisition. MK participated in the design of the study. All authors read and approved the final manuscript.

\section{Competing interests}

The authors declare that they have no competing interests.

Received: 18 March 2011 Accepted: 25 April 2012

Published: 25 April 2012

\section{References}

1. Ingels NB Jr: Daughters GT, 2nd, Stinson EB, Alderman EL: measurement of midwall myocardial dynamics in intact man by radiography of surgically implanted markers. Circulation 1975, 52(5):859-867.

2. Cheng A, Langer F, Rodriguez F, Criscione JC, Daughters GT, Miller DC, Ingels NB Jr: Transmural cardiac strains in the lateral wall of the ovine left ventricle. Am J Physiol Heart Circ Physiol 2005, 288(4):H1546-1556.

3. Osman NF, Kerwin WS, MCVeigh ER, Prince JL: Cardiac motion tracking using CINE harmonic phase (HARP) magnetic resonance imaging. Magn Reson Med 1999, 42(6):1048-1060.

4. Aletras AH, Ding S, Balaban RS, Wen H: DENSE: displacement encoding with stimulated echoes in cardiac functional MRI. J Magn Reson 1999, 137(1):247-252.

5. Zhong X, Spottiswoode BS, Meyer CH, Kramer CM, Epstein FH: Imaging three-dimensional myocardial mechanics using navigator-gated volumetric spiral cine DENSE MRI. Magn Reson Med 2010, 64(4):1089-1097.

6. Kindberg K, Karlsson M, Ingels NB Jr, Criscione JC: Nonhomogeneous strain from sparse marker arrays for analysis of transmural myocardial mechanics. J Biomech Eng 2007, 129(4):603-610.

7. Carlhall CJ, Nguyen TC, Itoh A, Ennis DB, Bothe W, Liang D, Ingels NB, Miller DC: Alterations in transmural myocardial strain: an early marker of left ventricular dysfunction in mitral regurgitation? Circulation 2008, 118(14 Suppl):S256-262.

8. Coppola BA, Omens $\mathrm{JH}$ : Role of tissue structure on ventricular wall mechanics. Mol Cell Biomech 2008, 5(3):183-196.

9. Kindberg K, Carlhall C, Karlsson M, Nguyen TC, Cheng A, Langer F, Rodriguez F, Daughters GT, Miller DC, Ingels NB Jr: Transmural strains in the ovine left ventricular lateral wall during diastolic filling. J Biomech Eng 2009, 131(6):061004.

10. McCulloch AD, Omens JH: Non-homogeneous analysis of threedimensional transmural finite deformation in canine ventricular myocardium. J Biomech 1991, 24(7):539-548.

11. Zhong $X$, Helm PA, Epstein FH: Balanced multipoint displacement encoding for DENSE MRI. Magn Reson Med 2009, 61(4):981-988.

12. Tsao J, Laurent D: N-SPAMM for Efficient Displacement-Encoded Acquisition in Myocardial Tagging. Proceedings of the 13th Annual Meeting of ISMRM: 2005 Miami Beach, FL, USA; 2005.

13. Stuber M, Spiegel MA, Fischer SE, Scheidegger MB, Danias PG, Pedersen EM, Boesiger P: Single breath-hold slice-following CSPAMM myocardial tagging. MAGMA 1999, 9(1-2):85-91.

14. Heiberg E, Sjogren J, Ugander M, Carlsson M, Engblom H, Arheden H: Design and validation of Segment-freely available software for cardiovascular image analysis. BMC Med Imaging 2010, 10:1.

15. Moon-Ho Song S, Napel S, Pelc NJ, Glover GH: Phase unwrapping of MR phase images using Poisson equation. IEEE Trans Image Process 1995, 4(5):667-676.

16. Farnebäck G, Rydell J, Ebbers T, Andersson M, Knutsson H: Efficient Computation of the Inverse Gradient on Irregular Domains. Computer Vision, 2007 ICCV 2007 IEEE 11th International Conference on: 2007 2007, 1-8, IEEE.

17. Waldman LK, Fung YC, Covell JW: Transmural myocardial deformation in the canine left ventricle. Normal in vivo three-dimensional finite strains. Circ Res 1985, 57(1):152-163.

18. Sigfridsson A, Haraldsson $H$, Ebbers $T$, Knutsson $H$, Sakuma $H$ : In vivo SNR in DENSE MRI; temporal and regional effects of field strength, receiver 
coil sensitivity and flip angle strategies. Magn Reson Imaging 2011, 29(2):202-208.

19. Moore CC, Lugo-Olivieri CH, McVeigh ER, Zerhouni EA: Three-dimensional systolic strain patterns in the normal human left ventricle: characterization with tagged MR imaging. Radiology 2000, 214(2):453-466.

20. Hess AT, Zhong X, Spottiswoode BS, Epstein FH, Meintjes EM: Myocardial 3D strain calculation by combining cine displacement encoding with stimulated echoes (DENSE) and cine strain encoding (SENC) imaging. Magn Reson Med 2009, 62(1):77-84

21. Kuijer JP, Marcus JT, Gotte MJ, van Rossum AC, Heethaar RM: Threedimensional myocardial strains at end-systole and during diastole in the left ventricle of normal humans. J Cardiovasc Magn Reson 2002, 4(3):341-351.

22. Ennis DB, Epstein FH, Kellman P, Fananapazir L, McVeigh ER, Arai AE Assessment of regional systolic and diastolic dysfunction in familial hypertrophic cardiomyopathy using MR tagging. Magn Reson Med 2003, 50(3):638-642

\section{Pre-publication history}

The pre-publication history for this paper can be accessed here: http://www.biomedcentral.com/1471-2342/12/9/prepub

doi:10.1186/1471-2342-12-9

Cite this article as: Kindberg et al:: Myocardial strains from 3D displacement encoded magnetic resonance imaging. BMC Medical Imaging 2012 12:9.

\section{Submit your next manuscript to BioMed Central} and take full advantage of:

- Convenient online submission

- Thorough peer review

- No space constraints or color figure charges

- Immediate publication on acceptance

- Inclusion in PubMed, CAS, Scopus and Google Scholar

- Research which is freely available for redistribution

Submit your manuscript at www.biomedcentral.com/submit 